Maria Salomé Martins Ferreira ${ }^{1}$

Maria da Graça Pereira ${ }^{2}$

${ }^{1}$ Escola Superior de Saúde, Instituto Politécnico de Viana do Castelo. Travessa da Fonte Quente 12, Meadela. 4900-895 Viana do Castelo Minho Portugal.

salomeferreira@ess.ipvc.pt ${ }^{2}$ Faculdade de Psicologia Universidade do Minho.

\section{O papel moderador do tipo de família na relação entre incapacidade funcional e qualidade de vida em doentes com lombalgia crônica}

\author{
The moderator role of family type in the relationship \\ between functional disability and quality of life \\ in patients with chronic low back pain
}

Abstract Chronic low back pain is a disease that interferes with quality of life and the patient's functional capability. This study aimed to identify the moderating effect of the "Type of Family" in the relationship between functional disability and quality of life in patients with chronic low back pain. Two hundred and three patients with low back pain for longer than 3 months participated in the study. The instruments used were: Medical Outcome Study (MOS 20); Roland Morris Disability Questionnaire (RMDO; Family Adaptability and Cohesion Evaluation Scales (FACES II). Regression analyses were performed in order to test the moderating effect of the type of family. The results showed that patients with lower levels of disability presented better quality of life in the intermediate and balanced families, and this relationship was even stronger in balanced families. According to the results, intervention programs in chronic low back pain, besides the patient, should include the family particularly in balanced families since they are the ones that feel the impact of the disease on their quality of life the most.

Key words Chronic low back pain, Disability, Family, Quality of life
Resumo A lombalgia crônica constitui-se como uma doença que interfere na qualidade de vida e na capacidade funcional do paciente. Este estudo teve por objetivo identificar o efeito moderador do "Tipo de Família" na relação entre a incapacidade funcional e a qualidade de vida em doentes com lombalgia crônica. Participaram no estudo 213 pacientes com lombalgia há mais de 3 meses. Os instrumentos utilizados foram: Medical Outcome Study (MOS-20); Roland Morris Disability Questionnaire (RMDQ); Family Adaptability and Cohesion Evaluation Scales (FACES II). Foram realizadas análises de regressão com o objetivo de testar o efeito moderador do tipo de família. Os resultados revelaram que os doentes com níveis mais baixos de incapacidade funcional apresentavam melhor qualidade de vida nas famílias intermédias e equilibradas, com relação mais forte nestas últimas. De acordo com os resultados, os programas de intervenção na lombalgia crônica, além do doente, devem incluir a família, particularmente nas equilibradas, que são as que mais sentem o impacto da doença na sua qualidade de vida.

Palavras-chave Lombalgia crônica, Incapacidade, Família, Qualidade de vida 


\section{Introdução}

A lombalgia crônica constitui-se como uma doença que interfere na qualidade de vida e na incapacidade funcional ${ }^{1}$. A família é a primeira unidade social na qual o indivíduo se insere e a doença crônica representa uma crise significativa para o doente e família ${ }^{2}$. Na lombalgia crônica, os indivíduos deparam-se com mudanças em todas as áreas de funcionamento pessoal e social e muitas pacientes apresentam alterações que levam a mudanças na sua qualidade de vida com exacerbação dos sintomas físicos e interferência nos esforços de reabilitação ${ }^{3,4}$.

A incapacidade funcional é normalmente definida como a restrição da capacidade do indivíduo para desempenhar atividades usuais da vida diária e serve para quantificar o impacto da doença ao nível das atividades básicas e instrumentais de vida diária ${ }^{5}$, papéis profissionais do individuo, papéis não ocupacionais e papéis no âmbito do lazer ${ }^{6}$. Ao nível das implicações físicas, nas doenças crônicas, a incapacidade funcional é um dos possíveis desfechos que provoca grande impacto negativo no bem-estar individual, gera maior necessidade de assistência, quer formal quer informal, e obriga o paciente a cuidados por longos períodos ${ }^{6}$.

Os indivíduos com doença crônica têm que enfrentar o desconforto e a incapacidade, seguir o tratamento de forma regular, modificar comportamentos para minimizar os resultados indesejáveis, ajustar sua vida social e o trabalho às suas limitações funcionais e ainda lidar com as consequências emocionais ${ }^{7}$. Frequentemente, o doente e a família não estão preparados para as alterações físicas e aquelas entre os períodos de crise e de estabilidade e para a incerteza do futuro $^{8}$. Assim, cada família tenta encontrar o seu estilo próprio de comunicação, as suas regras, as suas crenças, bem como a forma de manter a estabilidade ${ }^{9}$. A doença crônica representa uma crise significativa acarretando mudanças ao nível da estrutura e do processo ${ }^{10}$, nomeadamente ao das rotinas, na interação entre os seus elementos, nas relações interpessoais no sentido de se adaptarem à doença $\mathrm{a}^{11,12}$. Assim, perante a doença crônica, a família pode ser obrigada a redistribuir as tarefas entre seus outros membros, que até então não tinham essa responsabilidade ${ }^{13}$. De uma forma geral, quanto mais próximo o grau de parentesco maior será o impacto e a probabilidade dos familiares terem que modificar a sua vida em função dos cuidados ao doente ${ }^{14}$.
A literatura mostra duas dimensões chave no funcionamento familiar: a coesão e a adaptabilidade. A primeira refere-se aos vínculos e aos laços emocionais que os membros da família estabelecem entre si enquanto que a adaptabilidade é definida como a capacidade para a mudança ou flexibilidade do sistema familiar ${ }^{15}$. De acordo com o modelo referido, cada tipo de família tem as suas caraterísticas. Assim, nas famílias extremas existem dificuldades na coesão e adaptabilidade, em que o impacto da doença num dos seus elementos, poderá não acrescentar mais instabilidade; nas intermédias, os níveis de adaptabilidade e coesão são também eles intermédios; nas equilibradas existe grande capacidade de adaptabilidade às novas situações e proximidade emocional com respeito pela autonomia e identidade de cada membro, permitindo-lhes adaptar-se melhor à doença crônica, pois facilmente adotam estratégias de coping para esse efeito, nomeadamente a distribuição das regras e papéis de forma igualitária e democrática, sendo que estes são distribuídos proporcionalmente à idade de cada um.

O objetivo do presente estudo foi estudar o papel moderador do tipo de família na relação entre a incapacidade funcional e a qualidade de vida.

\section{Metodologia}

\section{Participantes}

O estudo incluiu uma amostra de 203 pacientes, com lombalgia há mais de 3 meses e que se encontravam a fazer tratamento de Fisioterapia (90) ou de Acupuntura (113) para a dor lombar. Os participantes apresentaram uma média de idade de 51.64 anos ( $\mathrm{DP}=14.13)$, maioritariamente do sexo feminino $(72,2 \%)$, casados ou em união de fato $(77,7 \%)$, com o curso superior completo $(39,3 \%)$, a trabalhar no setor terciário $(48,9 \%)$, a exercer a sua atividade profissional (60\%), com uma duração média de lombalgia de 69 meses $(\mathrm{DP}=9,63)$.

\section{Tipo de Estudo}

Trata-se de um estudo quantitativo, descritivo, transversal (um momento de avaliação no tempo) e correlacional, permitindo estabelecer relações entre as variáveis. 


\section{Material}

Sort-Form General Health Survey ${ }^{16,17}$. O MOS 20 é a forma reduzida do instrumento original (149 itens) e constitui-se como uma medida capaz de ter em conta a multidimensionalidade do conceito de Qualidade de Vida. O MOS 20 é uma escala composta por 20 questões sobre seis dimensões de qualidade de vida: funcionamento físico, funcionamento social, papéis, saúde mental, saúde geral e dor. Um resultado elevado indica melhor qualidade de vida. Na versão adaptada para a população portuguesa, o coeficiente de consistência interna foi respetivamente de 0,85 ; 0,87 e 0,$88 ; 0.84 ; 0,79$, e 0,83 para as seis escalas referidas, sendo o coeficiente da escala global de $0,89^{17}$. Na versão do estudo, o alfa de Cronbach foi de 0,90 para a escala total, de 0,84 para a subescala Funcionamento Físico; 0,72 para a subescala Desempenho de Papéis Sociais; 0,84 para a subescala Saúde Mental, e 0,87 para a subescala Perceção do Estado de Saúde, 0,86 para a subescala funcionamento social e 0,89 no item dores.

Roland Morris Disability Questionnaire $(\text { RMDO })^{18,19}$. O RMDQ foi inicialmente desenvolvido para medir a incapacidade física (baseado em autorrelato) em pacientes com lombalgia $^{19}$. Este instrumento permite avaliar as limitações físicas decorrentes de dor relatada na coluna lombar e é composto por 24 questões que descrevem a localização real da dor. Um resultado elevado indica maior incapacidade funcional. $\mathrm{O}$ instrumento apresenta boas características psicométricas ao nível da fidelidade e da validade ${ }^{20}$, em que o coeficiente alfa de Cronbach total da escala foi de 0,83 . Na presente amostra, o alfa de Cronbach foi de 0.84 e a adaptação portuguesa revelou um fator tal como na versão original ${ }^{19}$.

Family Adaptability and Cohesion Evaluation Scales (FACES II) ${ }^{21}$. Trata-se de um questionário que avalia o funcionamento familiar, permitindo a classificação das famílias segundo o Modelo Circumplexo. Esta escala foi desenvolvida para avaliar duas grandes dimensões da dinâmica familiar: a coesão e a adaptabilidade. Um resultado elevado indica uma coesão ou adaptabilidade familiar elevadas, respectivamente. Num estudo realizado em Portugal ${ }^{22}$ em adolescentes e suas famílias de acolhimento, os coeficientes de alfa de Cronbach foram 0,86 para a escala total, 0,77 para a subescala Coesão e 0,78 para a subescala Adaptabilidade. Na versão do estudo ${ }^{23,24}$, a escala total apresentou um alfa de 0,90 . No presente estudo, o alfa para a subescala Coesão foi de 0,83 e 0,84 para a adaptabilidade.

\section{Procedimento}

A primeira fase consistiu na identificação e seleção dos locais para a coleta de dados. Relativamente às clínicas de acupuntura e, pelo fato de esta profissão ainda não estar regulamentada em Portugal, foram selecionadas clínicas cujos acupunctores possuíssem a formação em Medicina Tradicional Chinesa e Acupuntura ministrada pela Associação Portuguesa de Acupuntura e Disciplinas Associadas, de forma a garantir a utilização dos mesmos procedimentos. Em relação às clinicas de fisioterapia, dado esta profissão estar regulamentada, este problema não se colocou.

Após a seleção das clínicas, foi enviado a cada diretor clínico, o pedido da autorização para a realização do estudo. Todos os pacientes preencheram os questionários de autorrelato, no final do quinto tratamento. No sentido dos doentes poderem ter recebido um número suficiente de tratamentos, foi escolhido o quinto qualquer que fosse a modalidade, dado o estudo avaliar a incapacidade funcional, devido à lombalgia, e a qualidade de vida.

Os critérios de inclusão foram: sofrer de lombalgia há mais de três meses; não estar a fazer tratamento para mais nenhuma situação patológica causadora de dor; ter idade superior a 20 anos; estar a receber tratamento de fisioterapia ou acupuntura sem acumular com outro tipo de cuidado para a lombalgia, com exceção de medicação para a dor. Todos os participantes que preenchiam os critérios de seleção foram convidados a participar no estudo. A participação dos pacientes foi voluntária, e todos o assinaram um consentimento informado. O período de coleta teve a duração de um ano.

\section{Análise de Dados}

Para o tratamento estatístico foi utilizado Statistical Package for Social Sciences (SPSS) versão 15.0, para Windows. Para a elaboração dos gráficos da moderação foi utilizado o software ModGraph ${ }^{25}$. Para descrever a amostra foi utilizada estatística descritiva. Ao nível das hipóteses, inicialmente começamos por verificar se existiam diferenças entre os grupos ao nível da variável “tipo de família”, mas dado que os grupos não se distinguem e os resultados obtidos para cada grupo (acupuntura e fisioterapia) serem os mesmos apenas com desvios mínimos, optamos por tratar os dados para a amostra global. Neste sentido, foram realizadas análises de regressão segundo o modelo linear múltipla (método enter), 
seguindo aquele proposto por Baron e Kenny ${ }^{26}$. Como a variável moderadora é nominal, para se calcular a interação foi necessário ponderar os efeitos desta variável preditora. Assim, enquanto o grupo em observação (e.g. as famílias extremas) recebe o valor 1 , o restante (famílias intermédias e equilibradas) recebeu como valor o negativo do rácio entre o número de casos do grupo em observação (número de famílias extremas) e o do restante (número de famílias intermédias e equilibradas), segundo a fórmula ${ }^{27}$ :

$$
c=-\frac{c 1}{c 2}
$$

$\mathrm{c} 1=$ (número famílias extremas)

c2 = (número famílias intermediárias e equilibradas)

\section{Resultados}

Efeito moderador do Tipo de Família Extrema na relação entre a Incapacidade Funcional e a Qualidade de Vida

A existência de interação $(\beta=0,124 ; p \leq 0,05)$ entre incapacidade funcional e tipo de família (extrema vs. não extrema) revela o efeito mode- rador exercido por este na relação entre incapacidade funcional e qualidade de vida (Tabela 1). Como se pode observar na Tabela 1 , não existe relação entre incapacidade funcional e qualidade de vida para as famílias extremas $(\beta=0,03 ; \mathrm{p}=$ $0,921)$, de modo que é apenas nas não extremas que doentes com níveis mais baixos de incapacidade funcional apresentam melhor qualidade de vida $(\beta=-0,53 ; \mathrm{p} \leq 0.001)$.

\section{Efeito moderador do Tipo de Família \\ Intermédia na relação entre a Incapacidade Funcional e a Qualidade de Vida}

A existência de interação $(\beta=0,188 ; p \leq 0,05)$ entre incapacidade funcional e tipo de família (intermédia vs. não intermédia) revela o efeito moderador exercido por este na relação entre incapacidade funcional e qualidade de vida (Tabela 2). Como se pode observar na Tabela 2 , a relação entre incapacidade funcional e qualidade de vida é mais fraca para as famílias intermédias $(\beta$ $=-0,41 ; \mathrm{p} \leq 0,001)$ que nas não intermédias $(\beta$ $=-1,05 ; \mathrm{p} \leq 0,001)$, embora em ambos os casos doentes com níveis mais baixos de incapacidade funcional apresentem melhor qualidade de vida.

Tabela 1. Resultados da moderação (Família Extrema) na relação entre a Incapacidade Funcional e Qualidade de Vida.

\begin{tabular}{|c|c|c|c|c|c|c|}
\hline \multirow{2}{*}{$\begin{array}{l}\text { Variáveis } \\
\text { Preditoras }\end{array}$} & \multicolumn{3}{|c|}{ Bloco I } & \multicolumn{3}{|c|}{ Bloco II } \\
\hline & $\beta$ std & $\mathbf{t}$ & sig & $\beta$ std & $\mathbf{t}$ & sig \\
\hline Incapacidade Funcional & $-0,497$ & $-8,254$ & 0,000 & $-0,494$ & $-8,259$ & $-8,259$ \\
\hline Tipo de Família (F. Extrema) & $-0,144$ & $-2,387$ & 0,018 & $-0,174$ & $-2,819$ & $-2,819$ \\
\hline Incapacidade Funcional x Família Extrema & & & & 0,124 & 2,023 & 2,023 \\
\hline $\mathrm{R}^{2}$ & & 0,277 & & & 0,269 & 0,269 \\
\hline $\mathrm{R}^{2}$ Ajustado & & 0,291 & & & 0,281 & 0,281 \\
\hline
\end{tabular}

Tabela 2. Resultados da Moderação (Família Intermédia) na relação entre a Incapacidade Funcional e Qualidade de Vida.

\begin{tabular}{|c|c|c|c|c|c|c|}
\hline \multirow{2}{*}{$\begin{array}{l}\text { Variáveis } \\
\text { Preditoras }\end{array}$} & \multicolumn{3}{|c|}{ Bloco I } & \multicolumn{3}{|c|}{ Bloco II } \\
\hline & $\beta$ std & $\mathbf{t}$ & sig & $\beta$ std & $\mathbf{t}$ & sig \\
\hline Incapacidade Funcional & $-0,507$ & $-8,392$ & 0,000 & $-0,651$ & $-6,977$ & 0,000 \\
\hline Tipo de Família (F. Extrema) & $-0,120$ & $-1,983$ & 0,049 & $-0,120$ & $-2,004$ & 0,046 \\
\hline Incapacidade Funcional x Família Extrema & & & & 0,188 & 2,016 & 0,045 \\
\hline $\mathrm{R}^{2}$ & & 0,270 & & & 0,263 & \\
\hline $\mathrm{R}^{2}$ Ajustado & & 0,285 & & & 0,274 & \\
\hline
\end{tabular}


Efeito moderador do Tipo de Família Equilibrada na relação entre a Incapacidade Funcional e a Qualidade de Vida

A existência de interação $(\beta=-0,160 ; \mathrm{p} \leq$ 0,01 ) entre incapacidade funcional e tipo de família (equilibrada vs. não equilibrada) revela o efeito moderador exercido por este na relação entre incapacidade funcional e qualidade de vida (Tabela 3). Como se pode observar na Tabela 3, a relação entre incapacidade funcional e qualidade de vida é mais forte para as famílias equilibradas $(\beta=-0,72 ; \mathrm{p} \leq 0,001)$ que nas não equilibradas $(\beta$ $=-0,38 ; \mathrm{p} \leq 0,001)$, embora em ambos os casos doentes com níveis mais baixos de incapacidade funcional apresentam melhor qualidade de vida.

\section{Discussão}

O tipo de família modera a relação entre a incapacidade funcional e a qualidade de vida. Doentes com níveis mais baixos de incapacidade funcional apresentam melhor qualidade de vida nas famílias intermédias e equilibradas, sendo esta relação mais forte nestas últimas.

O fato de os resultados desta investigação mostrarem que a relação entre a incapacidade funcional e a qualidade de vida é forte quando os doentes pertencem a uma família equilibrada, poderá ter a ver com o fato de num sistema familiar equilibrado os indivíduos terem a oportunidade de serem mais autónomos e também mais coesos e íntimos em relação uns aos outros ${ }^{11}$. Assim, o estressor dor crônica de um dos elementos e a sua incapacidade funcional pode ser sentida e vivenciada com maior intensidade por todos elementos da família, numa base de solidariedade e de afeto mútuo, refletindo-se na qualidade de vida de todos os elementos.
De fato, a família equilibrada é caracterizada pela proximidade emocional dos seus elementos, com respeito pela autonomia e identidade, bem como por fronteiras claras entre as gerações ${ }^{10}$. Pelo contrário e nesta linha de pensamento, em famílias extremas, com dificuldades na coesão e adaptabilidade, o impacto da doença neste caso de dor crônica num dos seus elementos, poderá não a destabilizar muito mais, que já não era coesa ou flexível, ao nível da qualidade de vida.

A lombalgia crônica restringe a capacidade do indivíduo para desempenhar as suas atividades da vida diária e gera um efeito negativo no bem-estar individual. Por conseguinte, esta situação pode levar a uma maior necessidade de cuidados por parte dos outros, nomeadamente dos elementos da famíliaa ${ }^{28}$. Num estudo em doentes com lombalgia crônica ${ }^{28}$, os autores verificaram alterações ao nível das limitações físicas que impediam os pacientes de fazerem tarefas domésticas, levando os cônjuges a realizá-las e a ocupar-se de novas atividades. Os cônjuges e, por vezes os filhos, assumem responsabilidades familiares anteriormente levadas a cabo pelo indivíduo que agora se confronta com a lombalgia. Os autores referem que, ao nível das consequências familiares, a lombalgia crônica provocava alteração dos papéis. Estas mudanças na família refletem-se na qualidade de vida, na incapacidade funcional e nas relações familiares ${ }^{29}$. Neste sentido, compreende-se o papel moderador da família na relação entre a incapacidade funcional e a qualidade de vida. Malheiro ${ }^{30}$ concluiu que a qualidade do ajustamento do casal moderava a relação entre a ansiedade e a qualidade de vida em doentes com fibromialgia. Por sua vez, Duarte ${ }^{31}$ encontrou a mesma relação em doentes com lúpus, verificando que quando o ajustamento do casal era baixo, se verificava uma relação preditiva entre a depressão e o domínio físico da qualidade de vida. Todos estes estudos enfatizam o papel da família ao nível da qualidade de vida.

Tabela 3. Resultados da moderação (Família Equilibrada) na relação entre a Incapacidade Funcional e Qualidade de Vida

\begin{tabular}{|c|c|c|c|c|c|c|}
\hline \multirow{2}{*}{$\begin{array}{l}\text { Variáveis } \\
\text { Preditoras }\end{array}$} & \multicolumn{3}{|c|}{ Bloco I } & \multicolumn{3}{|c|}{ Bloco II } \\
\hline & $\beta$ std & $\mathbf{t}$ & sig & $\beta$ std & $\mathbf{t}$ & sig \\
\hline Incapacidade Funcional & $-0,501$ & $-8,443$ & 0,000 & $-0,489$ & $-8,330$ & 0,000 \\
\hline Tipo de Família (F. Extrema) & 0,197 & 3,318 & 0,001 & 0,195 & 3,329 & 0,001 \\
\hline Incapacidade Funcional x Família Extrema & & & & $-0,160$ & $-2,732$ & 0,007 \\
\hline $\mathrm{R}^{2}$ & & 0,295 & & & 0,320 & \\
\hline $\mathrm{R}^{2}$ Ajustado & & 0,288 & & & 0,310 & \\
\hline
\end{tabular}


Se a família não conseguir dar resposta aos desafios impostos pela doença de um dos seus elementos e se não puder se reorganizar com vista ao seu equilíbrio, terá dificuldades na adaptação às necessidades e exigências do desenvolvimento psicológico dos seus membros ${ }^{32}$. A influência de fatores psicológicos, provocados pela doença de um elemento da família, vai interferir na qualidade de vida, uma vez que esta não avalia apenas a presença da doença ou a perspetiva do seu tratamento mas a experiência do indivíduo ao nível das suas necessidades e do seu bem-estar efetivo. Se as alterações provocadas pela doença crônica não conseguem ser superadas pelo apoio e reorganização das funções da família, vai ser mais difícil para o indivíduo doente superar as implicações da doença crônica.

A incapacidade funcional provoca grande efeito no bem-estar individual, gerando maior necessidade de assistência quer formal quer informal ao nível dos cuidadores, que muitas das vezes são os próprios familiares ${ }^{6}$, explicando a importância da família na relação entre a incapacidade funcional e a qualidade de vida. Quando a família é confrontada com novos problemas ou exigências, tenta manter o equilíbrio utilizando as capacidades e os recursos que possuía ${ }^{33}$. O tipo de família extrema tem mais dificuldade em manter o equilíbrio utilizando os seus recursos (estratégias de coping). Assim, o impacto da incapacidade funcional ao nível da qualidade de vida pode não ser sentido com tanta intensidade, como nas famílias equilibradas e intermédias, pois a extrema pode não ter capacidade de se reorganizar para lidar com a nova exigência da doença crônica ficando muitas vezes bloqueada e centrada nesta ${ }^{34}$.

Em suma, os resultados apontam para a necessidade dos profissionais de saúde considerarem o funcionamento familiar na abordagem do doente com lombalgia. A intervenção familiar deve ser uma opção que permita às famílias desenvolver competências para dar resposta aos desafios impostos pela doença, favorecendo assim a adaptação às necessidades e exigências do desenvolvimento psicológico dos seus membros ${ }^{32}$.

Futuras investigações deverão ter também em atenção a fase do ciclo de vida, uma vez que o impacto da doença na família vai depender da fase de desenvolvimento em que esta se encontra ${ }^{35}$.

\section{Limitações}

O estudo apresenta limitações, nomeadamente o fato de as medidas de avaliação serem instrumentos de autorrelato uma vez que estão sujeitas a problemas de interpretação e desejabilidade social. Outra limitação prende-se com o fato deste estudo ser descritivo e, por esse fato, não explicar se o tipo de família pode ter tido alguma influência no tipo de tratamento escolhido pelos doentes, i.e. fisioterapia ou acupuntura. Estudos futuros deveriam utilizar um design longitudinal e avaliar estes doentes, no final do tratamento e follow-up no sentido de perceber o impacto da lombalgia, na família, ao longo do tempo.

\section{Colaboradores}

MSM Ferreira trabalhou na concepção e redação do artigo, resultados e discussão. MG Pereira trabalhou na revisão crítica do artigo, metodologia e discussão dos resultados. 
Referências

1. Antunes RS, Macedo BG, Amaral TS, Gomes HA, Pereira LSM, Rocha FL. Pain, kinesiophobia and quality of life in chronic low back pain and depression. Acta Ortop Bras 2013; 21(1):27-29.

2. Pincus T. Effect of cognition on pain experience and pain behavior, Diathesis-stress and the causal conundrum. In: Young K, Nocholson A, editors. Psychological knowledge in Court. New York: Springer; 2006. p. 166193.

3. Katon W, Sullivan M. Depression and chronic medical illness. J Clin Psychiat 1990; 150(1):3-11.

4. Lamers L, Meerding WJ, Severens J, Brouwer W. The relationship between productivity and health-related quality of life: an empirical exploration in persons with low back pain. Qual Lif Res 2005; 14(3):805-813.

5. Jette AM. Disability trends and transitions. In: Binstock RH, George LK, editors. Handbook of Aging and the Social Science. San Diego; Academic Press: 1996. p. 94-116.

6. Lamb VL. A cross-national study of quality of life factors associated with patterns of elderly disablement. Soc Sci Med 1996; 42(3):363-377.

7. Virtuoso J, Jair S, Guerra RO. Incapacidade funcional em mulheres idosas de baixa renda. Cien Saude Colet 2011; 16(5):2541-2548.

8. McDaniel S, Hepworth J, Doherty W. Terapia Familiar Médica, em enfoque biopsicossocial com problemas de saúde. Porto Alegre: Artmed; 1994.

9. Enelow AJ, Forde DL, Brummel-Smith K. Entrevista Clínica e Cuidados ao Paciente. Lisboa: Climepsi Editores; 1999.

10. Olson D. Circumplex Model of Marital and Family Systems. J Fam Ther 2000; 22:144-167.

11. Minuchin S, Fishman HC. Técnicas de Terapia Familiar. Porto Alegre: Artmed; 2003.

12. Pratta E, Santos M. Família e adolescência: A influência do contexto familiar no desenvolvimento psicológico dos seus membros. Psicol Estud 2007; 12(2):247-256.

13. Martins A, Silva Y. Doenças Oncológicas e Representacões Sociais. Rev. Enf. Ref 2002; 9:11-24.

14. Viana V, Barbosa MC, Guimarães J. Doença Crônica na Criança, fatores Familiares e Qualidade de Vida. Psicologia, Saúde \& Doenças, 2007; 8(1):117-127.

15. Olson D, Gorall D. Circumplex Model of Marital and Family Systems. In: Walsh F, editor. Normal Family Processes. $3^{\text {a }}$ ed. New York: Guildford; 2003. p. 514-547.

16. Stewart AL, Hays RD, Ware JE. The MOS Short-Form Health Survey - reliability and validity in a patient population. Med Care 1998; 26(7):724-735.

17. Geada MJ. Perfil Psicopatológico e Qualidade de Vida nas Perturbações Distímicas e Depressão Major [dissertação]. Lisboa: Universidade Nova de Lisboa; 1996.

18. Roland M, Morris R. The Roland-Morris Disability Questionnaire and the Oswestry Disability Questionnaire. Spine J 2000; 25(24):3115-3124.

19. Ferreira MS, Pereira MG. Variáveis Psicológicas na Lombalgia Crônica: Um estudo com doentes em tratamento de Fisioterapia e Acupuntura [tese]. Braga: Universidade do Minho; 2009.

20. Perreault K, Dionne CE. Patient-physiotherapist agreement in low back pain. Clin J Pain 2005; 6(12):817-828.
21. Olson DH, Portner J, Bell RQ. FACES II: Family Adaptability and Cohesion Evaluation Scales. Minnesota: Family Social Science, University of Minnesota; 1993.

22. Fernandes OM. Família e emigração: um estudo da estrutura e do funcionamento familiar de uma "população" não migrante do conselho de Chaves e de uma "população” portuguesa no Cantão de Genebra 1994-1995 [dissertação]. Coimbra: Faculdade de Psicologia e de Ciências da Educação; 1995.

23. Tribuna, M. F. Famílias de acolhimento e vinculação na adolescência 1999-2000 [dissertação]. Coimbra: Instituto Superior Miguel Torga; 2000. Texto Policopiado.

24. Sardá JJ, Nicholas MK, Pimenta CAM, Asghari A, Corrêa CF, Oswaldo JJ. Validade e fidedignidade do Questionário Roland Morris de incapacidade em uma população de brasileiros com dor crônica. Rev Dor 2006; 7(4):892-904.

25. Jose PE. ModGraph-I: A programme to compute cell means for the graphical display of moderational analyses:The internet version, Version 3.0. Victoria University of Wellington, Wellington, New Zealand. 2013. [acessado 2013 dez 11]. Disponível em: http://pavlov. psyc.vuw.ac.nz/paul-jose/modgraph/

26. Kenny D. Moderator Variables. [on line] 2009. [acessado 2013 abr 11]. Disponível em: http://davidkenny.net/ $\mathrm{cm} /$ moderation.htm

27. Aitken LS, West SG. Multiple Regression: Testing and Interpreting interactions. Newbury Park: Sage; 1991.

28. Lorig K, Ritter P, Stewart AL, Sobel DS, Brown BW, Bandura A, Gonzalez VM, Laurent DD, Holman HR. Chronic disease self management program, 2-year health status and health care utilization outcomes. Med. Care 2001; 39(11):1217-1223.

29. Strunin L, Boden L. Family Consequences of Chronic Back Pain. Soc. Sci. Med 2004; 58(1):385-393

30. Malheiro M. Variáveis psicossociais na Fibromialgia: Um estudo de casal [dissertação]. Universidade do Minho; 2009.

31. Duarte SMS. Variáveis Individuais e Familiares no Lúpus Eritematoso Sistémico [dissertação]. Braga: Universidade do Minho; 2008

32. Ferros L. Jovens, drogas e famílias - Uma breve Revisão da Literatura. Toxicodependências 2003; 9:71-83.

33. MacCubbin HI, Patterson JM. The family stress process: The double ABCX model of adjustment and adaptation. Marria. Fam. Reve 1993; 6(7):7:37.

34. Steinglass P. Multiple family discussions groups for patients with chronic medical illness. Fam. Syst. Health 1998; 16(1/2):55-70.

35. Pereira MG. A Perspectiva bio psicossocial na Avaliação em Psicologia da Saúde, Modelo Interdependente. Psi. Teo. Prat 2002; 7(2):183-191.

Artigo apresentado em 09/07/2014

Aprovado em 12/05/2015

Versão final apresentada em 14/05/2015 